\title{
INTERPRETACIÓN DEL HECHO METROPOLITANO EN ZARAGOZA
}

\author{
$M^{a}$ Yolanda SÁNCHEZ DEGANO \\ Departamento de Geografía y Ordenación del Territorio \\ Universidad de Zaragoza
}

\begin{abstract}
Resumen: En el contexto de las nuevas dinámicas territoriales que han venido definiendo el fenómeno urbano en las últimas décadas, la ciudad de Zaragoza está siguiendo un proceso de crecimiento expontáneo hacia la periferia ocupando sucesivamente su área circundante. Este hecho está dando lugar a una realidad urbana que ha adquirido una nueva dimensión al desbordar los límites municipales; una realidad cuyas características creemos que nos permiten hablar del fenómeno metropolitano en nuestra ciudad. Partiendo de esta idea se ha llevado a cabo una propuesta de delimitación en un intento por definir la supuesta "Area Metropolitana de Zaragoza", en cualquier caso no comparable al resto de las españolas.
\end{abstract}

Palabras clave: Area metropolitana, difusión e integración, espacio-funcional.

\begin{abstract}
In the context of the new territorial dynamics, which have defined the urban phenomenon in the last decades, the city of Zaragoza is suffering an spontanous growing process outwards to the outskirts, occupying its surrounding area. This fact is determining an urban reality with a new dimension as it has overflowed the municipal borders; a reality whose characteristics allow us to talk about the metropolitan phenomenon in our city. Beginning from this idea, a delimitation proposal has been carried out in an attempt to define the supposed "Zaragoza's metropolitan area", non comparable however to the other Spanish ones.
\end{abstract}

Key words: Metropolitan, space-funtional spread and integration.

\section{INTRODUCCION}

El área central del denominado "Corredor del Ebro", como parte fundamental de la estructura territorial en Aragón, ha adoptado en el contexto actualmente definido por las nociones de "cohesión" e "integración" espacio-funcional (CASTAÑER, 1992, 1994), un significado mucho más amplio que invita a reflexionar sobre el fenómeno metropolitano en Zaragoza. 
Las nuevas dinámicas territoriales, vinculadas básicamente a los fenómenos de descentralización-periurbanización generadas desde la capital han derivado en la formación de un espacio cuyas características generales permiten referirnos al cambio sufrido por Zaragoza de "ciudad nodal" a "ciudad difusa".

Sin embargo adoptando una perspectiva mucho más flexible, enmarcada en el proceso de intensificación creciente de las interdependencias entre núcleos de población materializadas en un territorio cada vez más amplio, se puede incluso llegar a hablar de "metrópoli", y por lo tanto referirnos a la existencia de un área metropolitana, lo que en definitiva equivaldría a detectar la consolidación de los sistemas de interrelaciones espacio-funcionales en el denominado "Corredor del Ebro" (SÁNCHEZ, 1996).

Teniendo en cuenta estas consideraciones, si en primer lugar asumimos la presencia de un espacio metropolitano, el siguiente paso sería establecer sus posibles límites no olvidando que estos siempre serán por definición difusos y variables en el tiempo y en el espacio; aspectos en los que nos vamos a centrar a lo largo de estas líneas.

\section{INTERPRETACION DEL HECHO METROPOLITANO EN ZARAGOZA}

Tal y como dejan constancia algunas de las definiciones relativas al hecho metropolitano (DE ESTEBAN, 1981; ESTEBAN 1991; JONES, 1992; BARRERO, 1993), está claro que solamente derivado de la integración en un territorio de las relaciones entre los distintos núcleos de población y la ciudad central, en base a sus funciones y potencialidades, es posible hablar del fenómeno metropolitano en el sentido más estricto de la palabra.

Trasladando el concepto básico de área metropolitana al ámbito aragonés, la existencia de una ciudad central (Zaragoza) de la que "dependen", desde un punto de vista funcional, toda una serie de municipios que a la vez se conectan entre sí, configurando un área definida por un sistema de interrelaciones que gravita en tomo a 1 municipio central, son aspectos que puede llegar a justificar el "hecho metropolitano" en Zaragoza. Pero en realidad, ¿dónde se fijan los límites de dicha área metropolitana?, ¿qué municipios la integran?, ¿estamos ante una verdadera área metropolitana o por el contrario se trata de un tipo de aglomeración simple o área urbana sin más?.

Encontrar las respuestas correctas a estas preguntas es una labor ardua y difícil puesto que la definición de las áreas metropolitanas así como su delimitación, ya sea 
desde la óptica etimológica, administrativa, legal, funcional y no digamos geográfica, ha demostrado ser hasta hoy una tarea bastante problemática y un tanto imprecisa.

Efectivamente, después de varias décadas de estudio y propuestas de modelos de concepción metropolitana se ha puesto de manifiesto que el término "área metropolitana" lleva implícito multitud de connotaciones que dejan en evidencia su complejidad, en tanto en cuanto el hecho urbano, ya sea en sus aspectos cualitativos como cuantitativos, es un proceso, por naturaleza, contínuo y dinámico en el tiempo y en el espacio que impide designar criterios standard para su definición, puesto que no existe una sóla realidad urbana ni un sólo modelo universal, sino varias realidades metropolitanas acordes con un desarrollo urbano concreto.

En este estado de cosas, hasta hoy no se ha llegado a determinar ningún acuerdo internacional o mundial común para la definición y delimitación de las áreas metropolitanas, siendo esta una de las principales razones que justifica la disparidad de criterios utilizados según el momento y los autores. No obstante, de forma genérica e indistintamente se han venido aplicando una serie de criterios que podemos denominar "tipo", dando como resultado conclusiones coherentes siempre y cuando se han ajustado a las peculiaridades y rasgos diferenciales de la realidad de cada área.

Sobre la base de estas consideraciones, la interpretación del hecho metropolitano en Zaragoza pasa de manera obligada por la revisión de algunas referencias teóricas, donde merecen especial atención ese conjunto de criterios "tipo"; criterios que en cualquier caso están íntimamente asociados a las nuevas dinámicas territoriales que han venido definiendo el fenómeno urbano en las últimas décadas.

\section{REFERENCIAS TEORICAS: CRITERIOS DE DELIMITACION}

La doctrina sobre el fenómeno metropolitano tiene su origen a comienzos de siglo en E.E.U.U. De forma oficial el concepto terminológico ("directrices metropolitanas", "Zona Metropolitana") se recoge en el censo de la Oficina Federal de los E.E.U.U. (1.910), entendiendo como tal aquella que tiene una ciudad central con más de 200.000 habitantes, no incluida en el radio de acción de otro núcleo de población, y que comprende toda parte del territorio situada a una distancia menor de 10 millas del centro, siempre que tenga una densidad de población múnima de 150 habitantes por milla cuadrada $\left(60 \mathrm{hab} . / \mathrm{km}^{2}\right)$; una definición como vemos fundamentada exclusivamente en un criterio de tipo demográfico (BERRY et al., 1968; FONT, 1972; DE ESTEBAN, 1981). 
A partir de aquí, se fueron produciendo constantes variaciones derivadas de la revisión e introducción de diferentes variables, siempre en la línea de precisar un poco más la definición y delimitación de las áreas metropolitanas, hasta llegar, cuarenta años más tarde, a determinar modelos apoyados en el concepto teórico de "sistema integrado de funciones". Surgieron así las figuras S.M.A. (Standard Metropolitan Area) (1.950) y de las S.M.S.A. americanas (Standard Metropolitan Statistical Area) (1.960) que, a pesar de sus muchas críticas, han sido la referencia básica de gran parte de los estudios y análisis realizados sobre áreas metropolitanas en Norteamérica y Europa (NUNES I ALONSO, 1986; SERRA, 1991).

Junto a estos, los sucesivos modelos surgidos de las críticas derivaron en la aparición de nuevos criterios que en definitiva no han hecho nada más que añadir matices al concepto, si bien en líneas generales todos ponen el énfasis en los aspectos de integración económico-funcional (FOX KARL, 1964; FRIEDMAN et al., 1965; BERRY, 1973; FONT, 1972; COOMBES et al., 1978, 1979; HALL y HAY, 1980) lo que en última instancia nos lleva a la conclusión de que la delimitación de la metrópoli debe hacerse en función de "intensidades metropolitanas" (SERRA, 1991), es decir a partir de las relaciones de carácter funcional y por lo tanto del grado de interrelación entre el núcleo principal y los secundarios.

Llegados a este punto, esta claro que de la comparación entre España y E.E.U.U. se deduce fácilmente que la realidad urbana española dista mucho de 1 a norteamericana, de modo que cualquier intento de aplicación de modelos como los aquí expuestos, ha de hacerse en su justa medida, adaptándose obligatoriamente a los rasgos característicos del fenómeno urbano en nuestro país.

Con varios años de retraso en la incorporación española a las nuevas dinámicas territoriales, la expresića "área metropolitana" en España se recoge legalmente por primera vez en la Ley 121/1.963, de 2 de diciembre (Boletín Oficial del Estado, 5-1263) referido al Planeamiento del Area Metropolitana de Madrid (redacción del nuevo Plan General de Ordenación). No obstante parece ser que dicha expresión todavía no es adecuada y tanto en la Reforma de la Ley del Suelo y Ordenación Urbana 1/9/1.975 de 2 de mayo, como en la Ley de Bases del Estatuto de Régimen Local 41/1.975 de 19 de noviembre, se refieren a un concepto jurídico similar que se corresponde con el de "Entidad" ("Entidad Local", "Entidad Urbanística Especial", "Entidad Municipal Metropolitana"); hecho que en definitiva evidencia las deficiencias terminológicas y conceptuales relativas a la definición y delimitación de "areas metropolitanas" en España (ligadas a la complejidad del término en sí), en tanto que todavía no llega a justificarse razonadamente lo que se entiende por "área metropolitana" además de no hacer alusión alguna a los criterios de delimitación. 
Sin embargo, al margén de lo estrictamente jurídico, fueron los organismos oficiales españoles los que recogieron con mayor amplitud la expresión, haciendo mención al concepto y criterios utilizados para su delimitación; concretamente y en primer lugar por parte de la Dirección General de Urbanismo del Ministerio de la Vivienda, cuando se publicó el estudio titulado "Areas Metropolitanas Españolas en 1.960" (revisión efectuada en 1.967) y después por el III Plan de Desarrollo Económico y Social (monografía Desarrollo Regional y Estructuras y Seroicios Urbanos).

Dichos organismos llegaron al concepto y delimitación de "áreas metropolitanas" apoyándose en los argumentos y planteamientos surgidos de los estudios realizados en los países más avanzados, de tal modo que para la definición de las mismas se hizo uso de criterios similares a los referidos con anterioridad', siendo las características demográficas las que en principio resultaron ser más importantes ${ }^{2}$.

Con posterioridad el III Plan de Desarrollo Económico y Social recoge además, como aspecto destacado para la definición de las mismas, el desarrollo económico de los municipios que la integran, seleccionando una serie de criterios referidos, en primer lugar, a la existencia de un mercado mínimo de trabajo diversificado en un entorno geográfico contínuo (umbral mínimo 20.000 trabajadores); y en segundo término, a 1 a especialización mínima de la mano de obra determinada a partir de coeficientes de especialización por sectores.

A partir de aquí, siguiendo en la misma línea que caracteriza los modelos de los países anglosajones, mucho más especializados en estos temas, fue el anterior Ministerio de Obras Públicas, Transporte y Medio Ambiente (M.O.P.T.M.A), en e1 marco del Plan Director de Infraestructuras (P.D.I.) y nuestros investigadores los que a través de las nuevas aportaciones, derivadas de sus estudios, han ido incorporando otros criterios que, en último término, no han hecho sino evidenciar una vez más la complejidad y arbitrariedad que, derivado de la variedad de criterios propuestos, existe a la hora de definir y delimitar dichas áreas.

En cualquier caso, los estudiosos del tema mantienen, de forma generalizada, I a aplicación de los más significativos, que pueden agruparse en los siguientes puntos:

\footnotetext{
1 En concreto, la Dirección General de Urbanismo del Ministerio de la Vivienda estableció de manera oficial (no con carácter legislativo) los criterios de delimitación de las "Areas Metropolitanas" españolas según los criterios tipo fijados por el profesor Kingsley Davis, de la Universidad de California (Berkeley).

2 Se definieron como Areas Metropolitanans aquellas que presentasen un municipio central con una población superior a los 50.000 habitantes; alcanzasen un total de 100.000 habitantes en el conjunto del área, constituida por una serie de municipios que cumpliesen: una densidad de población mínima de $100 \mathrm{hab} / \mathrm{km}^{2}$, un índice de crecimiento demográfico mínimo, entre 1.930 y 1.960 , del 152\% (15\% decenal acumulativo); y formasen un continum urbano con el municipio central.
} 
a) Consideraciones de carácter demográfico: tamaño, dinámica poblacional y densidad demográfica .

b) Consideraciones relativas a la ocupación del suelo y morfología urbana: continuum urbano, en convivencia con los usos agrícolas del suelo que puede poner en contacto físico diferentes unidades administrativas.

c) Consideraciones relacionadas con la especialización funcional: primacía de las actividades productivas, terciario y terciario superior o cuaternario.

d) Consideraciones sobre la interrelación de los núcleos: mantenimiento de un sistema cotidiano de relaciones sociales y económico-funcionales.

e) Consideraciones de vinculación y dependencia funcional con respecto al núcleo central.

De acuerdo con estos planteamientos, llegamos a la conclusión de que la esencia más simple del concepto de "área metropolitana" se fundamenta en tres aspectos básicos: tamaño poblacional de los núcleos, primacía de un núcleo central de carácter abierto y difuso, e integración económica y social cristalizada en un sistema de relaciones cotidianas; todos ellos, aspectos que, en última instancia, están enmarcados en el contexto de las actuales dinámicas territoriales.

\section{El AMBITO METROPOLITANO EN ZARAgOZA. PROPUESTA DE DELIMITACION}

Tal y como ya aludíamos al inicio de este artículo a propósito de 1 a consideración o no del hecho metropolitano en Zaragoza, la primera aproximación al tema nos llevaba a la conclusión de que la presencia de Zaragoza como gran ciudad central alrededor de la que gravita un conjunto de municipios conectados entre sí, en función de un sistema de relaciones socio-funcionales, era una de las razones principales que nos permitía hablar del fenómeno metropolitano en Zaragoza.

No obstante desde un principio parecía que el interés de la cuestión estaba más en el hecho de determinar, una vez convencidos de que el fenómeno metropolitano podía ser asumible, si realmente estábamos ante una verdadera área metropolitana en todas sus dimensiones, comparable por ejemplo a la de Barcelona o Madrid, o si simplemente se trataba de una área urbana de menor envergadura. 
Para despejar esta incógnita el paso previo antes de precipitarnos a cualquier tipo de consideración, era intentar definir funcional y espacialmente el área en cuestión; es decir, proponer una delimitación a partir de una serie criterios "tipo" adaptados a nuestra realidad urbana, sin olvidar que, ni en este ni en ningún otro caso, el hecho de delimitar supone una definición estricta, en tanto que como unidad espaciofuncional no se trata de una realidad estática.

En ese intento por prefijar los criterios que nos aproximasen a la delimitación de esta presumible "Area Metropolitana de Zaragoza", se hacía inevitable dar un repaso a la bibliografía y revisar los diferentes estudios que confirmaban la existencia fáctica, pero no legal, de un área metropolitana en nuestra ciudad.

Los primeros trabajos que aluden a ella en estos términos, datan de la década de los sesenta. Se trata de los documentos oficiales que fueron eleborados por la Dirección General de Urbanismo y por el III Plan de Desarrollo Económico y Social, anteriormente mencionados, donde Zaragoza se encuentra entre las primeras áreas metropolitanas que para España habian sido definidas de forma oficial a efectos estadísticos, y a efectos de desarrollo económico y social.

Por otro lado fue el Ministerio de Obras Públicas y Transporte a través de algunas de sus publicaciones, el segundo organismo público que ha hecho alusión a Zaragoza como Area Metropolitana, siendo dos los documentos que se han consultado: en primer lugar el realizado por el C.E.O.T.M.A. (Centro de Estudios de Ordenación del Territorio y Medio Ambiente) con el título La problemática inherente al papel de los sistemas de infraestructuras en la conformación de las áreas metropolitanas de desarrollo reciente. Aplicación al Area Metropolitana de Valencia (1.982-83); y en segundo término, el documento elaborado por el M.O.P.T.M.A. en el marco del Plan Director de Infraestructuras (P.D.I.) para el período 1.993-2.007.

Por su parte, y a pesar de que la cuestión metropolitana en nuestra Comunidad Autónoma es un tema que en numerosas ocasiones ha pasado a un segundo plano, sobre todo en aquellos momentos en los que han surgido conflictos y vaivenes de carácter político $^{3}$, la Diputación General de Aragón, que ve en la creación institucional de la misma una necesidad "primaria" con el fin de coordinar conjuntamente los planes

\footnotetext{
3 Existen diversidad de opiniones sobre la viabilidad real o no de crear un área metropolitana en Zaragoza. A pesar de que son muchos los que están a favor de la misma, hay quienes han vertido opiniones más bien dubitativas acerca de esta cuestión. Para ello argumentan que dicha creación podría por un lado aumentar el sentimiento de postergación de los demás municipios zaragozanos y aragoneses respecto a esta banda de desarrollo que es el Corredor del Ebro donde se integra el Area Metropolitana; mientras que por otro, aluden a que suponiendo que los municipios que integrasen el área estuvieran dispuestos a asumir este compromiso, no cabe duda de que no existiría igualdad de condiciones entre estos y el municipio central a la hora de conceder las mismas oportunidades en el seno del nuevo organismo de gobiemo metropolitano, dado el carácter macrocéfalo de la capital.
} 
urbanos entre sí y con la ordenación del territorio de Aragón, también se ha planteado como objetivo prioritario, dentro del marco de la Ordenación del Territorio en Aragón, lo que se ha denominado"el diseño del Area Metropolitana de Zaragoza", que según algunas de sus publicaciones, comprendería al menos 26 municipios organizados en los cuatro ejes radiales con centro en la capital ${ }^{4}$.

Sobre la base de estos planteamientos, y teniendo como únicas referencias disponibles para profundizar acerca del tema las anteriormente mencionadas, nuestro objetivo, como ya se señaló, es intentar una nueva aproximación a la definición del área metropolitana de Zaragoza.

Con una base metodológica propia de cualquier método "clásico" de delimitación, el primero de los pasos dado fue la elección de unos criterios adecuados a las características que definen el entorno de la capital, para con posterioridad proceder a la superposición y aplicación de los mismos, delimitando finalmente el área.

El proceso de selección de dichos criterios nos llevó necesariamente a considerar, desde el principio, los que han sido definidos como criterios "tipo" para la delimitación de un Area Metropolitana, agrupándolos en aspectos de carácter demográfico, ocupación del suelo y morfología urbana, especialización funcional, e interrelación y vinculación de los núcleos.

Una vez repasados y teniendo en cuenta otros ejemplos de delimitación (FONT, 1972; DE ESTEBAN, 1981; JUNTA DE ANDALUCIA, 1985; NUNES I ALONSO, 1986; SERRA, 1991) se seleccionaron y combinaron cuatro de los indicadores más representativos de este tipo de áreas, definiendo unos umbrales de relevancia adaptados a una realidad urbana caracterizada por la disfuncionalidad territorial entre la capital y su entorno, consecuencia directa del carácter de mononuclearidad de Zaragoza, lo que nos obligó a prescindir de los umbrales medios aplicados de forma generalizada, en tanto que Zaragoza no es precisamente el prototipo de área metropolitana como pueda serlo la de Barcelona o Madrid. Estos son los siguientes:

1.- Densidad de población de cada municipio: mayor o igual a $50 \mathrm{hab} / \mathrm{km}^{2}$.

2.- Incremento poblacional de cada municipio entre 1.960-1.991: igual o superior al $10 \%$.

\footnotetext{
${ }^{4}$ En este sentido la D.G.A. ha elaborado una serie de estudios (Avance de Directrices de Ordenación Territorial y Urbanística) en los que se analiza de forma pormenorizada y por separado cada uno de los "ejes de expansión del entorno de Zaragoza", como paso previo a la definición de los criterios que perfilan la delimitación del Area Metropolitana de Zaragoza.
} 


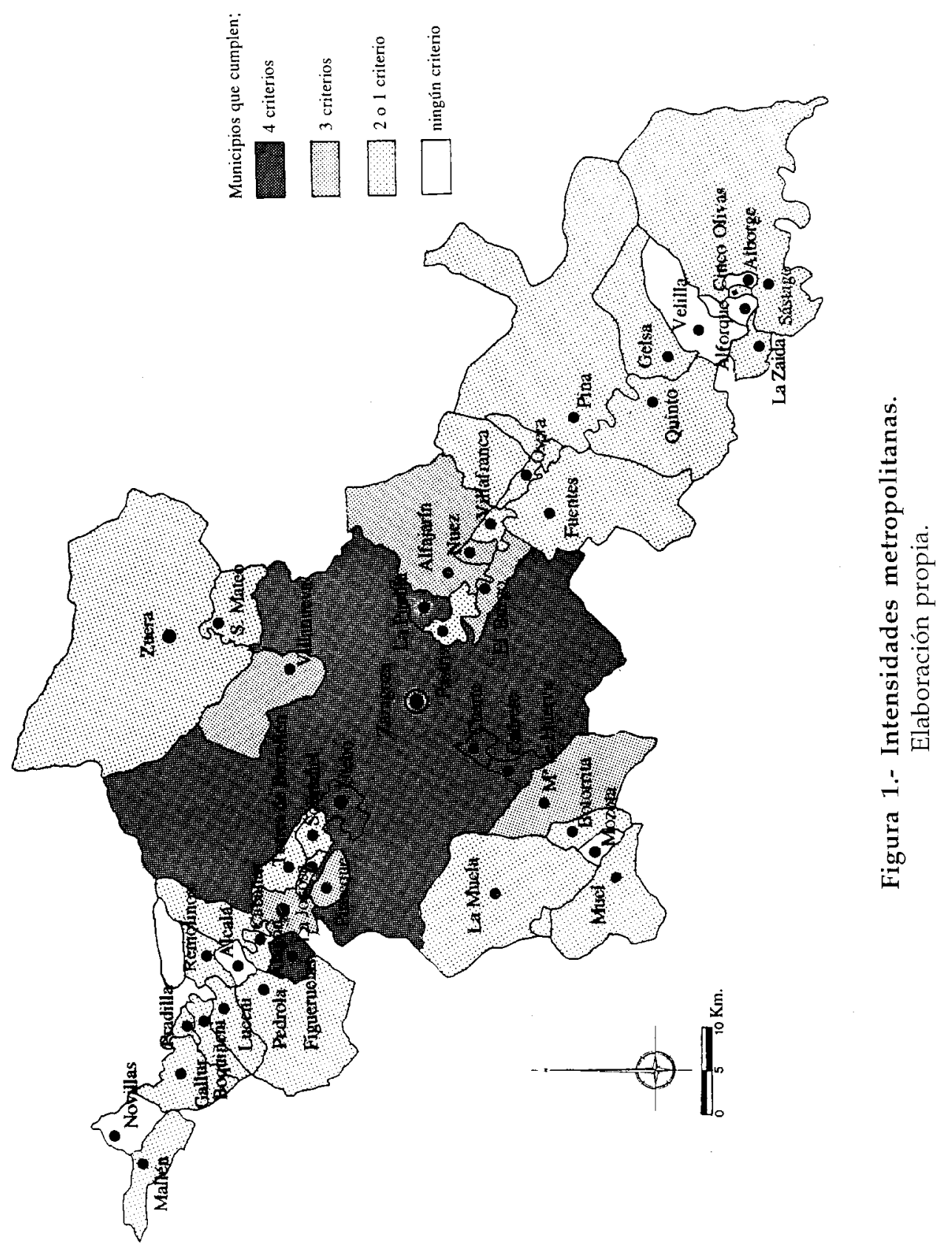


3.- Proporción de ocupados en puestos de trabajo no agrícola: superior o igual a 1 $75 \%$ del total.

4.- Intensidad de relación por motivos de trabajo (movilidad diaria): valor mínimo de desplazamientos obligados por motivos de trabajo 5\%.

La aplicación simplificada de estos indicadores al conjunto de municipios pertenecientes al área del denominado "Corredor del Ebro" (46 en total), dió como resultado una clasificación de los municipios de mayor a menor intensidad "metropolitana" en función del número de criterios que cumpliesen.

Se diferenciaron así tres niveles gradacionales (Fig. 1): una primera área central más intensa formada por cinco municipios (Utebo, Figueruelas, Cuarte, Cadrete y La Puebla de Alfindén) que cumplen simultáneamente los cuatro criterios seleccionados. Se trata de un espacio casi perfectamente contínuo sobre las carreteras de entrada/salida de Zaragoza (dirección Barcelona, Logroño y Valencia), salvo el municipio de Figueruelas que es el único que queda ligeramente separado del resto ${ }^{5}$, dentro de un radio de unos $12 \mathrm{~km}$ a la capital (compredidos en la isocrona de 15') a excepción del ya mencionado que se sitúa a $30 \mathrm{~km}$ (segunda isocrona de 15' a 30').

En el segundo nivel de "metropolitaneidad" (tres condiciones) donde se sigue la expansión territorial de Zaragoza a lo largo de los ejes principales de comunicación hasta una distancia de aproximadamente $30 \mathrm{~km}$ desde el centro. En este se incluyen los municipios de Villanueva de Gállego, Alfajarín, Nuez de Ebro, El Burgo, María de Huerva, Pinseque y Alagón, núcleos con una especialización funcional basada, al igual que los anteriores, en la industria.

Finalmente, el tercer nivel (dos o un critrerio) comprende muncipios contíguos a los grupos anteriores acabando de completar los ejes elementales de crecimiento y difusión de la capital. Por lo general se trata de núcleos de población de carácter industrial, aunque de menor significación que los anteriores, salvo excepciones; y de carácter residencial con escasa participación en el desarrollo industrial generado a instancias de la gran ciudad, vinculados funcionalmente a los niveles superiores.

Tras la aplicación de estos indicadores, de forma complementaria se utilizaron tres más, a los que sumamos información adicional recogida a través de trabajo de

5 El caso de Figueruelas tiene su explicación clara en la localización de la única gran empresa industrial con repercusiones espacio-funcionales a todos los niveles (local, comarcal, provincial y regional), concentrando cerca de 9.000 puestos de trabajo en un municipio que tan sólo cuenta con 867 habitantes, lo que genera un destacado porcentaje de desplazamientos diarios ("conmuting"), que le convierten en el segundo nodo de centralidad más importante después de Zaragoza. 
campo, con la finalidad de ajustar dichos límites, además de confirmar la validez de los primeros resultados obtenidos. Dichos indicadores hacen referencia a:

1.- La existencia o no de contigüidad espacial.

2.- Los puntos de ruptura en las intensidades de tráfico.

3.- La expansión de las residencias de carácter principal o secundario.

Hasta aquí, atendiendo a la totalidad de los indicadores expuestos y una vez valorados en su conjunto, la delimitación del área metropolitana que proponemos (Fig. 2) y las características de la misma son las siguientes (Tabla 1):

1-. El "Area Metropolitana de Zaragoza", comprendería un total de 17 municipios, exceptuando la capital. Ocupa una superfice de $723 \mathrm{~km}^{2}\left(1.782 \mathrm{~km}^{2}\right.$ si contamos con el municipio de Zaragoza), con una población que asciende a los 29.891 habitantes de hecho, más los 622.371 del municipio de Zaragoza (según datos del Censo de 1991), y una densidad de población de $366 \mathrm{hab} / \mathrm{km}^{2}$.

2-. El área está constituida por los municipios pertenecientes a los dos primeros niveles de intensidades "metropolitanas" (cumplimiento de 3 y 4 criterios). No obstante, municipios como los de Pastriz, Torres de Berrellén, Sobradiel, La Joyosa y La Muela (cumplimiento de 2 indicadores), se han admitido en el área por razones de posición respecto al resto de los municipios, es decir, por quedar envueltos totalmente por los núcleos que resultan incluidos en el área.

3-. Dentro de esta área, el municipio de Figueruelas en el eje NW queda algo desligado del "continutum urbano" (N-232). No osbtante esta ruptura física a la que aludimos, no es tal si hacemos referencia a la continuidad de flujos económicos ("continuum funcional") que subyacen en la base de los asentamientos industriales que, en este y en el resto de los ejes, supone un movimiento diario de capital, producción y mano de obra.

A la luz de esta propuesta de delimitación, y en función de los rasgos que definen este espacio, lo realmente claro es que no estamos ante un área de las características de la de Barcelona o Madrid donde solamente la población de los municipios que las constituyen, exceptuando la ciudad central, suponen más del $50 \%$ de la misma, cuando en nuestro caso no llega al $5 \%$ del total $(8,74 \%$ si consideramos conjuntamente los municipios del área y los barrios pedáneos pertenecientes a Zaragoza). Una realidad que viene definida por el fuerte desequilibrio poblacional que existe como consecuencia del gran tamaño demográfico del municipio de Zaragoza, que sólo en su ciudad ya concentra a casi el $92 \%$ del total de la población del área propuesta (595.236 
habitantes). Un área en la que no se superan $\operatorname{los} 366 \mathrm{hab} / \mathrm{km}^{2}$, frente a los más de 3.000 y $4.000 \mathrm{hab} / \mathrm{km}^{2}$ de las Areas Metropolitanas de Madrid y Barcelona; donde, a diferencia de nuestro caso, la intensificación y complejidad de los flujos de relaciones socio-funcionales supera el ámbito del Area Metropolitana por lo que hemos de referirnos a ellas como ciudades-región o Regiones Metropolitanas policéntricas, además de tener carácter de metrópolis internacionales.

Tabla 1.- Rasgos generales del "Area Metropolitana" propuesta.

\begin{tabular}{|l|c|c|c|c|c|c|}
\hline Municipio & Población & $\Delta$ Pob. & Superficie & Densidad & $\%$ Poplación no & \% Desplaz \\
\hline & $\mathbf{1 9 9 1}$ & $\mathbf{1 9 6 0 - 9 1}$ & $\mathbf{K m}^{2}$ & $\mathbf{H a b} / \mathbf{k m}^{2}$ & agrícola & \\
\hline Utebo & 7.766 & 236,60 & 17,7 & 438,76 & 93,9 & 73,74 \\
\hline Figueruelas & 867 & 132,57 & 17 & 51 & 86,7 & 79,08 \\
\hline Cuarte & 1.369 & 284,62 & 8,9 & 153,82 & 96,4 & 86,9 \\
\hline Cadrete & 909 & 138,57 & 11,9 & 76,39 & 86,5 & 83,5 \\
\hline Puebla Alf. & 1.439 & 116,33 & 17 & 84,65 & 89,1 & 64,26 \\
\hline Villanueva & 2.460 & 115,11 & 76 & 32,37 & 86 & 66,49 \\
\hline Pinseque & 1.367 & 102,78 & 16,1 & 84,91 & 87,3 & 58,84 \\
\hline Alagón & 5.487 & 104,12 & 24,2 & 226,74 & 90,8 & 19,46 \\
\hline Ma Huerva & 809 & 118,97 & 108,1 & 7,48 & 86,6 & 73,05 \\
\hline El Burgo & 1.235 & 104,22 & 24,9 & 50 & 90,8 & 32,14 \\
\hline Alfajarín & 1.546 & 132,36 & 137,6 & 11,24 & 89,7 & 43,96 \\
\hline Nuez Ebro & 535 & 99,07 & 8,2 & 65,24 & 81,5 & 25,7 \\
\hline Sobradiel & 597 & 88,97 & 12,1 & 49,34 & 82,8 & 71,49 \\
\hline La Joyosa & 341 & 72,09 & 6,5 & 52,46 & 66,3 & 33,3 \\
\hline T. Berrellén & 1.438 & 81,33 & 53,8 & 26,73 & 83,2 & 43,75 \\
\hline La Muela & 978 & 96,83 & 166,1 & 5,45 & 80,4 & 68 \\
\hline Pastriz & 748 & 81,13 & 16,5 & 45,33 & 82,2 & 55,8 \\
\hline \hline Total & 29.891 & 127,39 & 722,6 & 41,3 & 89,2 & 79,82 \\
\hline \hline Municipio Central & & & \\
\hline \hline Zaragoza & 622.371 & 190,72 & 1059 & 587,70 & 98,5 & 17 \\
\hline \hline
\end{tabular}

* : Desplazamientos hacia Zaragoza y entre los municipios del "Corredor del Ebro".

Fuente: Censos de población 1961, 1991. Edt.Instituto Nacional de Estadística. Madrid.

Elaboración propia. 


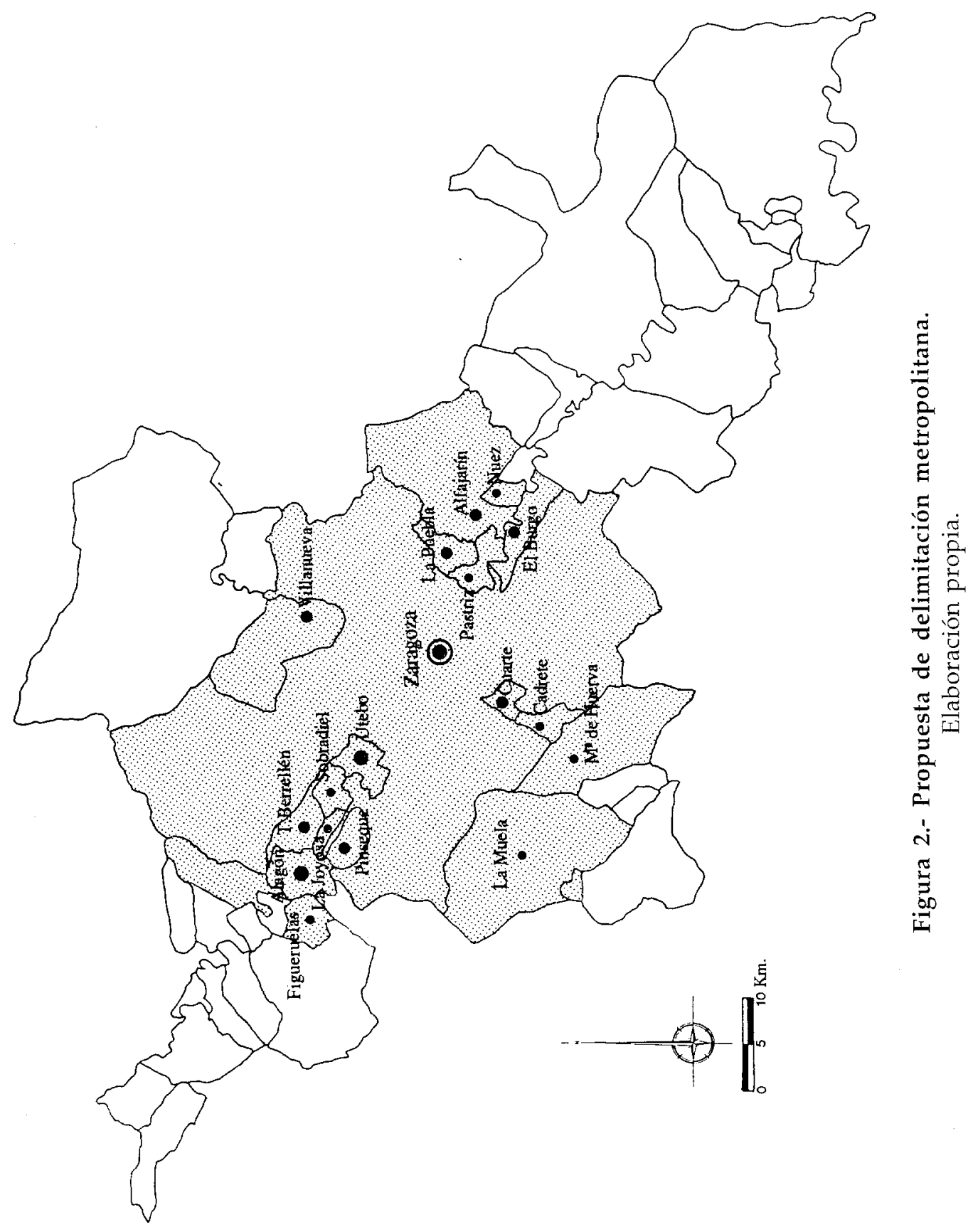


Con estas referencias, y si tenemos en cuenta su morfología, la intensidad de las relaciones funcionales, la diferencia extrema entre el tamaño de población de la ciudad central y el resto de los municipios, y la densidad de población, la primera conclusión a la que llegamos es que Zaragoza define un área mucho menos compleja que las descritas por Barcelona o Madrid; un área que a nuestro entender tampoco es equiparable a la de Valencia o el "Gran Bilbao", a pesar de que en el P.D.I. (Plan Director de Infraestructuras) estén categorizadas en el mismo nivel.

Por supuesto, con ello no pretendemos negar la afirmación a la que llegábamos a 1 inicio de este epígrafe cuando hablábamos de la posibilidad de asumir el fenómeno metropolitano en Zaragoza, ni tampoco que dicha área no pueda calificarse como Area Metropolitana real, un hecho comúnmente aceptado entre los investigadores del tema y la administración pública de la Comunidad Autónoma, que aboga por la creación legal e institucional de esta figura como elemento esencial en la planificación y ordenación territorial. Sin embargo, sobre la base de los datos aportados y dadas las características que la definen, se hablaría con más propiedad si nos refiriésemos a ella como "área metropolitana simple", o incluso si le diéramos el apelativo de "aglomeración urbana simple" o "área urbana" sin más.

No obstante, independientemente de la denominación que le asignemos, lo incuestionable es que, como consecuencia de los mecanismos y procesos dinámicos de difusión y expansión territorial vinculados a la reestructuración económica, Zaragoza, desbordando sus límites administrativos, se ha proyectado hacia su periferia sobre la base de una red principal de carreteras de carácter radial, hasta una corona de aproximadamente $15-30 \mathrm{~km}$ de radio, impulsando el desarrollo socio-económico de municipios próximos a ella, dando lugar así, a la configuración de una unidad espaciofuncional integrada mediante flujos y relaciones de interdependencia; es decir, una estructura reticular abierta y dinámica, determinada por la intensidad de las conexiones.

\section{BIBLIOGRAFIA}

BARRERO RODRIGUEZ, M C. (1993): Las Areas Metropolitanas. (Inst. García Oviedo).Civitas. Universidad de Sevilla.

BERRY, B..J.L.., GOHHEN, P.G. \& GOLDSTEIN, H. (1968): Metropolitan area definition: a recoaluation of concept and statistical practice. Washington D.C., U.S. Bureau of the Census.

BERRY, B..J.L. (1973): Growth centers in the American Urban Systems, Vol. 2, Cambridge, Massachussets, Ballinger.

BORJA, J. (1986): "Las áreas metropolitanas de la España actual". Rev. Alfoz. Monografía. Gobierno Metropolitano, 33, 20-23. Madrid. 
BORJA, J. (1991): "Grans ciutats i àrees metropolitanes a Europa. El cas de Barcelona". El fet metropolità: Interpretacions geogràfiques. 9-14. Institut d'Estudis Metropolitans de Barcelona. Barcelona.

CASTAÑER, M. (1994): "La ciudad real en Cataluña. Las áreas de cohesión". Rev. Ciudad y Territorio. Estudios Territoriales. 94, 101-114. M.O.P.T.M.A. Madrid.

CASTAÑER, M. et al. (1993): "La ciudad funcional en Catalunya. Las áreas de cohesión". XIJI Congreso Nacional de Geografía. 345-349. A.G.E. Universidad de Sevilla.

CASTAÑER, M. y RIERA, P. (1995): "Las áreas urbanas y la articulación del territorio catalán". Rev. Situación. 3, 235-248. Servicio de estudios B.B.V. Bilbao.

CEOTMA. (1982-1983): La problemática inherente al papel de los sistemas de infraestructuras en la conformación de las áreas metropolitanas de desarrollo reciente. Aplicación al área metropolitana de Valencia. Centro de Estudios de Ordenación del Territorio y Medio Ambiente. Madrid.

COMUNIDAD DE MADRID (1990): Madrid Región Metropolitana: Estrategia Territorial y Actuaciones. Arpegio. Madrid.

COOMBES, M.G. et al. (1978): "Towards a more rational consideration of census areal units: daily urban systems in Britain". Environment and Planning, 10, 1179-1185.

COOMBES, M.G. et al (1979): "Daily urban systems in Britain: from theory to practice" Environment and Planning, A. vol. II. 565-574.

CORVINOS, P. y HERRERO, J. (1995): "Hacia un modelo organizativo para la ordenación territorial de Zaragoza y su espacio metropolitano". Il Congreso sobre Ordenación del Territorio. Departamento de Ordenación Territorial, Obras Públicas y Transportes. Gobiemo de Aragón. Zaragoza.

DE ESTEBAN, A. (1981): Las áreas metropolitanas en España: Un análisis ecológico. Centro de Investigaciones sociológicas. Colección "Monografías". Madrid.

DIPUTACION GENERAL DE ARAGON.(1991) Avance de directrices de Ordenación Territorial y Urbanística. Prograna de gestión urbanistica en el Eje de Desarrollo Norte del entorno de la ciudad de Zaragoza. Departamento de Ordenación Territorial, Obras Públicas y Transportes. Gobierno de Aragón. Zaragoza (Inédito).

DIPUTACION GENERAL DE ARAGON (1991): Avance de directrices de Ordenación Territorial y Urbanistica. en el Eje de Desarrollo Oeste de Zaragoza. Departamento de Ordenación Territorial, Obras Públicas y Transportes. Gobierno de Aragón. Zaragoza. (Inédito).

DIPUTACION GENERAL DE ARAGON (1991): Avance de directrices de Ordenación Territorial y Urbanística. Programa de gestión urbanística en el Eje de Desarrollo Este del entomo de la ciudad de Zaragoza. Departamento de Ordenación Territorial, Obras Públicas y Transportes. Gobierno de Aragón. Zaragoza. (Inédito).

ESTEBAN, J. (1991): "El fet metropotità". El fet metropolità: Interpretacions geogràfiques. 15-30. Institut d'Estudis Metropolitans de Barcelona.

FERRER, M. y CALVO, J.J. (1993): "El comportamiento de la España metropolitana en la década de los ochenta". XIII Congreso Nacional de Geografía. A.G.E. Universidad de Sevilla. 
FONT, A. (1972): "La delimitación de las áreas metropolitanas: el caso de Barcelona". Cuadernos de Arquitectura y Urbanismo, 87.

FOX, KARL A. (1964): Integrating National and Regional Models for Economic Stabilization and Growth. University of Pittsburg.

FRIEDMAN, J.\& MILLER, J. (1965): The Urban Field. Journal of de American Institute of Planners.

HALL, P. y HAY, D. (1980): Growth centers in the Urban Europ System. Educational Books. Heinemann. Londres.

JONES, E. (1992): Metropolis. Las grandes ciudades del mundo. Alianza Editorial. Madrid.

JORDANA DE POZAS, L. (1967): "Las Areas Metropolitanas en España y en el extranjero". Problemas de Urbanismo Moderno. IEAL. Madrid.

JUNTA DE ANDALUCIA (1989): El Area Metropolitana de Sevilla. Consejería de Obras Públicas y Transportes (Gabinete de Estudios Metropolitanos). Sevilla.

LACLETA ALMOLDA, S. (1994): "Reflexiones en torno a la mejora de la gestión de los espacios urbanos-metropolitanos: Una visión actualizada desde el punto de vista de la coordinación interadministrativa". Revista Aragonesa de Administración Pública. Departamento de Presidencia y Relaciones Institucionales. Gobierno de Aragón. Zaragoza.

M.O.P.T.M.A. (1994): Plan Director de Infraestructuras (PDI). Modelo Territorial y Estrategias. Secretaría de Estado de Política Territorial y Obras Públicas. Dirección General de Planificación Territorial. Madrid.

NUNES I ALONSO, J. (1986) "Aproximació a l'estructura de l'area metropolitana de Barcelona: les estructures d'intensitat diària". Documents d'Anàlisi Geogrâfica. 8-9, 71-90. Publicaciones del Departamento de Geografía. Universidad Autónoma de Barcelona.

SANCHEZ DEGANO, Y. (1994): "Delimitación del área metropolitana de Zaragoza a partir de los movimientos pendulares industriales. Fuentes y Método de trabajo". IX Jornadas sobre Metodología de la investigación científica sobre fuentes aragonesas. Instituto de Ciencias de la Educación. Universidad de Zaragoza.

SANCHEZ DEGANO, Y. (1996): La red urbana del Corredor del Ebro. Dpto de Geografía y Ordenación del Territorio. Universidad de Zaragoza (Tesis Doctoral inédita).

SERRA, J. (1991): "La ciutat metropolitana delimitacions, desconcentracions, desequilibris". El fet metropolità: Interpretacions geogràfiques, 31-52. Institut d`Estudis Metropolitans de Barcelona. Barcelona. 